\title{
Total sleep time and kynurenine metabolism associated with mood symptom severity in bipolar disorder
}

\author{
Dahlia Mukherjee $^{1}$ (D) | Venkatesh Bassapa Krishnamurthy ${ }^{1,2}$ | Caitlin E. Millett ${ }^{1,3}$ | \\ Aubrey Reider $^{1}$ | Adem Can ${ }^{4}$ | Maureen Groer ${ }^{5}$ | Dietmar Fuchs ${ }^{6}$ | \\ Teodor T Postolache ${ }^{4,7,8}$ | Erika F. H. Saunders ${ }^{1,9}$
}

${ }^{1}$ Department of Psychiatry, Penn State Milton S Hershey Medical Center, Hershey, PA, USA

${ }^{2}$ Sleep Research and Treatment Center, Penn State Milton S Hershey Medical Center,

Hershey, PA, USA

${ }^{3}$ Department of Neural and Behavioral Sciences, Penn State University, Hershey, PA, USA

${ }^{4}$ University of Maryland School of Medicine, Baltimore, MD, USA

${ }^{5}$ University of South Florida, School of Nursing, Tampa, FL, USA

${ }^{6}$ Innsbruck Medical University, Innsbruck, Austria

${ }^{7}$ Rocky Mountain MIRECC (Mental Illness Research Education and Clinical Center), Denver, CO, USA

${ }^{8}$ The Military and Veteran Microbiome Consortium for Research and Education (MVM-Core), Denver, CO, USA

${ }^{9}$ Department of Psychiatry, University of Michigan Medical School, Ann Arbor, MI, USA

\section{Correspondence}

Dahlia Mukherjee, Department of Psychiatry, Penn State Milton S Hershey Medical Center, Hershey, PA, USA.

Email: dmukherjee@pennstatehealth.psu.edu

Funding information

The Penn State Clinical \& Translational Research Institute; Pennsylvania State University CTSA; NIH/NCRR, Grant/Award Number: UL1RR033184
Objective: Chronic, low-level inflammation is associated with symptomatic bipolar disorder (BD) and with chronic insomnia. Disrupted sleep is a feature of episodes of both mania and depression. We examined the effect of neopterin, a marker of cellular immune activation, and kynurenine (KYN), an inflammatory byproduct of the serotonin pathway, on the association between total sleep time and depression severity in BD. Method: Twenty-one symptomatic BD participants and 28 healthy controls $(\mathrm{HC})$ were recruited and followed during usual clinical care. At baseline and after symptomatic recovery, total sleep time was objectively measured with actigraphy for 1 week and blood plasma was collected to measure the serotonin precursor tryptophan (TRP), KYN, the KYN/TRP ratio, and neopterin levels. Statistical analyses were conducted using chi-square, independent $t$ tests and hierarchical linear multiple regression models.

Results: Total sleep time was correlated positively with depressive severity and negatively with manic severity. TRP was significantly reduced in BD participants compared to $\mathrm{HC}$. KYN, TRP, and the KYN/TRP ratio were associated with depressive severity when total sleep time and body mass index (BMI) were included in the model. The KYN/TRP ratio trended towards a negative association with mania symptoms, controlling for BMI and total sleep time, in acutely symptomatic BD participants. Neopterin was not associated with sleep or mood severity. After usual clinical care, BD participants showed significantly decreased clinical symptoms but no significant differences in sleep phenotype or biomarkers.

Conclusion: Inflammation, sleep, and mood are closely intertwined. Future research into the effect of inflammation on sleep in BD may lead to clinical markers of outcome.

KEYWORDS

inflammation, kynurenine pathway, sleep

\section{1 | INTRODUCTION}

Bipolar disorder (BD) is a severe psychiatric disorder, with a complex and sometimes heterogeneous range of symptoms. ${ }^{1} \mathrm{BD}$ is one of the leading causes of disability, demonstrating, on average, a less favorable prognosis than major depressive disorder (MDD). ${ }^{2}$ As a clinically heterogeneous syndromic disorder, the biological changes in BD have been reported in depressive and manic episodes, and any unifying biological underpinnings of this disorder have remained elusive. ${ }^{3-5}$ Research shows that BD presents with numerous immune-inflammatory and 
sleep abnormalities. ${ }^{6-8}$ Activation of the kynurenine (KYN) pathway has been supported in a number of psychiatric disorders, including $\mathrm{BD}^{9,10}$.While many studies have independently explored sleep, ${ }^{11-13}$ inflammation, ${ }^{7,14}$ and the $\mathrm{KYN}^{9,15}$ pathway in $\mathrm{BD}$, there is increasing evidence that inflammation is an important factor in mood, stress, and sleep disturbance ${ }^{16,17}$ and needs to be examined simultaneously for a better understanding of the underlying biological mechanisms. Additionally, there is a dearth of research exploring inflammation, metabolites of the KYN pathway, or sleep in acutely ill BD patients. ${ }^{4}$

Mood spectrum disorders (BD, recurrent depressive disorder, and seasonal affective disorder) are accompanied by sleep dysregulations that are particularly common among bipolar participants. ${ }^{8,12,13,18}$ Sleep dysregulations in mood disorders commonly occur in acute manic or depressive states but are also prevalent during remission. ${ }^{8,19-21}$ A metaanalysis focusing on sleep in remitted BD participants highlights disturbances in several sleep parameters: longer sleep latency, longer sleep duration, and poorer sleep efficiency. ${ }^{21}$ Sleep is a remarkably sensitive, but not specific, indicator of distress, and dysregulated sleep can occur for a variety of reasons. Inflammation has been shown to be associated with sleep disturbance and objective short sleep in primary insomnia and obstructive sleep apnea, and may mediate the association between sleep apnea and adeposity. ${ }^{22-24}$ By examining the association of sleep dysregulation with inflammatory biomarkers in $\mathrm{BD}$, we may gain an understanding of the sleep, mood, and inflammation connection.

The essential amino acid tryptophan (TRP) is a substrate for two important biosynthetic pathways: the serotonin (5-hydroxytryptamine [5-HT]) pathway and the KYN pathway (Figure 1). During stress, proinflammatory cytokines activate the KYN pathway and deprive the serotonin pathway of TRP, thereby reducing serotonin synthesis. The dynamics of this process are characterized best by the kynurenine-to-TRP (KYN/TRP) ratio. One of the inflammatory drivers of this diversion of serotonin to $\mathrm{KYN}$ is neopterin, a catabolic product of guanosine triphosphate that is a marker of cellular immune system activation produced by macrophages upon stimulation by interferon-gamma. ${ }^{25-28}$ Increased peripheral KYN results in increased central KYN. KYN is further broken down into quinolinic acid, which is a potent agonist at the N-methyl-D-aspartate (NMDA) receptor, causing excitotoxic effects through receptor overactivation ${ }^{29,30}$ and inhibiting glutamate uptake, causing elevated extracellular glutamate concentrations. $^{31}$

In the current study, we investigated the relationship between markers of the KYN pathway, neopterin, sleep, and clinical symptoms in acutely ill BD participants. We were also interested in exploring biomarkers and sleep patterns after symptom remission, to determine whether these biomarkers were potentially state vs trait characteristics independent of mood state. We hypothesized that:

1. Patients with acute $B D$ would have significantly lower total TRP and a higher KYN/TRP ratio and neopterin levels than healthy controls (HC).

2. The KYN/TRP ratio and neopterin would be associated with severity of depressive and manic symptoms in acutely symptomatic participants with BD.

3. Sleep duration and biomarkers including kynurenine pathway metabolites (TRP, KYN/TRP) and the proinflammatory cytokine neopterin would be associated with depressive and manic symptom severity.

Finally, we were interested in whether these markers changed with symptom remission in asymptomatic patients, and explored differences in clinical symptoms, sleep, and biomarkers between baseline and follow up.

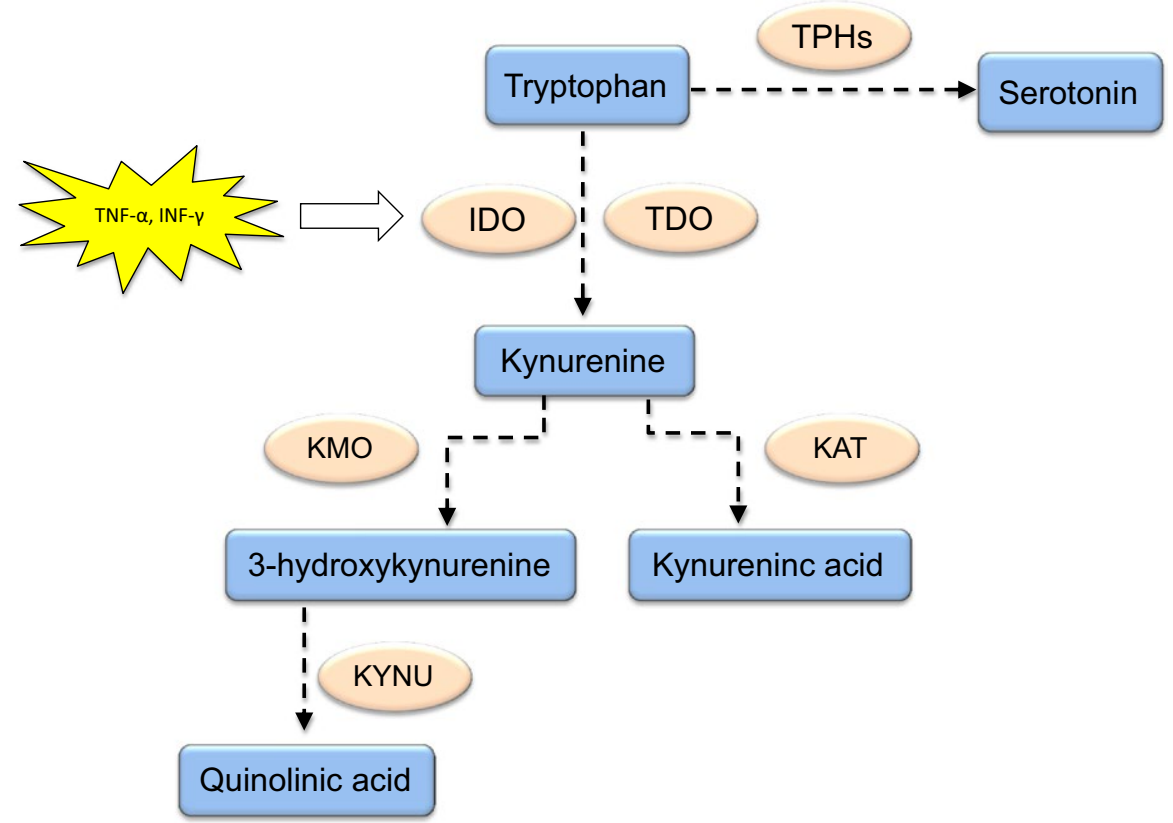

FIGURE 1 The kynurenine pathway. INF- $\gamma$, interferon-gamma; TNF- $\alpha$ tumor necrosis factor-alpha; TPH IDO, indolamine-2,3-dioxygenase; KAT, kynurenine aminotransferase; KMO, kynurenine 3-monooxygenase; KYNU, kynureninase; TDO, tryptophan-2,3dioxygenase; TPH, tryptophan hydroxylase 


\section{2 | PARTICIPANTS AND METHODS}

\section{1 | Participants}

Thirty BD participants with current mood symptoms participated in the study, and 21 completed all measures for this analysis. Participants were recruited when presenting for inpatient or partial hospital care at the Pennsylvania Psychiatric Institute, under Institutional Review Board Protocol No. 39164EP and NIH Office of Human Subject Research Exemption \#11509 (7/16/2012). Participants were screened and consented for the study, and assessments including sleep and biomarkers were administered while they were receiving inpatient or partial hospital care. Inclusion criteria for BD participants included adults with a primary diagnosis of BD I or II. Exclusion criteria included use of nonsteroidal anti-inflammatory drugs (NSAIDs) on a daily basis, active substance intoxication or withdrawal, major endocrinological or rheumatological illness, or pregnancy. Twenty-eight HC participants were recruited via advertisement, based on the inclusion criteria that they had no personal or family history of mental illness, rheumatological or endocrinological illness, pregnancy, or daily NSAID use. HCs provided actigraphy data in their home environment, while clinical and blood measures were collected at the research site.

\subsection{Measures}

Structured clinical interview based on the Mini Neuropsychiatric Interview $^{32}$ were conducted and current mood state was assessed using the Hamilton Depression Rating Scale-21 plus atypical items (HDRS-21+AT), ${ }^{33}$ and the Clinician-Administered Rating Scale for Mania (CARS-M). ${ }^{34}$ A combination of clinically significant manic and depressed symptoms defined a mixed-manic phenotype. HDRS$21+$ AT scores between 0 and 6 indicated no depression; scores between 7 and 17 indicated mild depression; scores between 18 and 24 indicated moderate depression; and scores over 24 indicated severe depression. CARS-M scores below 7 were considered non-manic. Scores between 7 and 12 were mild, and above 12 were severe manic phenotypes. The body mass index (BMI) for each participant was calculated by measuring height and weight. Participants received usual clinical care and were discharged from the program. After discharge from the hospital or partial hospital program, participants were followed each week by telephone and assessed for clinical improvement. A return visit was scheduled to collect a second blood sample when the subject was asymptomatic, or after 3 months had elapsed. Additional measures were collected but not used for this analysis. Saunders et $\mathrm{al}^{35}$ provided a more detailed report on the methods and results of analysis of fatty acid concentrations.

\section{3 | Objective measure of total sleep time}

During the period of initial evaluation, participants wore an actigraph (Sleepwatch-O, Ambulatory Monitoring, Inc., Ardsley, NY, USA), a watch-like device that measures acceleration, on the nondominant hand for 7 days. They also maintained a sleep log, which, combined with the actigraphy recording, provides an accurate assessment of objective sleep measures. ActiLife V.6.4.5 software (ActiGraph Software Department, Pensacola, FL, USA) was used to compute the actigraphy sleep variables, including total sleep time, bedtime variability, sleep latency, sleep duration, and sleep efficiency. BD participants completed the actigraph during hospitalization, and HCs completed it at home. Owing to the small sample size, we limited the analysis of sleep variables to the investigation of total sleep time only, which allowed us to conserve statistical power. However, an additional exploratory analysis of mean lag (variability in bedtime from night to night) was also made.

\subsection{Sample collection and biochemical analysis}

Participants fasted for at least 6 hours, and blood was drawn in vacutainers containing ethylenediaminetetraacetic acid (EDTA) in the morning. BD participants had blood drawn between 07:15 and 10:30 (average time 08:24) and HC participants had blood drawn between 08:00 and 14:00 (average time 09:19). After centrifugation for 10 minutes at $654.03 \mathrm{~g}$, the plasma supernatant was transferred to plastic tubes and maintained at $-80^{\circ} \mathrm{C}$ until processed. Total TRP (micromoles per liter) and KYN (micromoles per liter) were measured by reverse-phase high-performance liquid chromatography, as previously described ${ }^{36,37}$, using 3-nitro-L-tyrosine as an internal standard. The KYN/TRP ratio was calculated (expressed as micromoles of KYN per millimoles of TRP). Neopterin was measured by enzyme-linked immunosorbent assay (BRAHMS, Hennigsdorf, Germany) according to the manufacturer's instructions, with a detection limit of $2 \mathrm{nmol} / \mathrm{L}$.

\section{5 | Data and statistical analysis}

SPSS version 22 (IBM SPSS Statistics, IBM Corporation, Armonk, NY, USA) was used for all statistical calculations. Values of continuous variables were compared between the $\mathrm{BD}$ and $\mathrm{HC}$ groups using independent-samples $t$ tests, and categorical variables were compared using the Pearson chi-square test. Paired $t$ tests were used for baseline and follow-up measurements. In an exploratory analysis, Pearson's $r$ was used for separate bivariate correlations on demographics and clinical symptoms, sleep, and inflammation variables in the BD and $\mathrm{HC}$ samples. Baseline depression was predicted using linear regression models based on a priori variables, controlling for BMI due to the known association between inflammation and obesity. ${ }^{38}$ Model 1 included total sleep time and KYN/TRY, model 2 included total sleep time and KYN, model 3 included total sleep time and TRP, and model 4 had total sleep time and neopterin as predictor variables. The same models were implemented to predict baseline manic symptoms controlling for BMI. Paired $t$ tests were conducted to compare symptoms, total sleep time, and inflammatory variables from baseline to followup. However, follow-up data were available for considerably fewer participants. This was the result of an inability to maintain regular contact with some participants owing to a number of issues (e.g., unstable housing conditions). The maximum number of days to follow-up was 187; the median was 22 days and the average was 52 days. However, 
an analysis of dropouts vs those who continued to be a part of the study did not show any significant differences based on baseline clinical symptoms and demographics, including age and gender ( $P$ values $>$.16). Dependent variables were compared between BD smokers and nonsmokers using independent $t$ tests.

\section{3 | RESULTS}

\subsection{Demographic and clinical description of the sample}

A cohort of 30 participants with BD was recruited while in a symptomatic mood episode. Six participants did not have sleep assessment data, and three did not have biomarker data; therefore, 21 BD participants were included in the final analysis. A parallel HC group of 31 participants was recruited for the study, of which three participants did not have sleep data $(n=28)$. Participants with BD did not differ from the HC group in age, gender, or race (Table 1). A significantly higher proportion of participants with BD were unemployed, not married, and smokers than in the $\mathrm{HC}$ group (Table 1). Independent $t$ tests were run between the smokers and nonsmokers within the BD group, to detect any differences based on age, BMI, HDRS, CARS-M, TRP, KYN, KYN/TRP, and sleep variables. No differences were detected, with all $P$ values above .10 .

Clinically, BD individuals had a significantly higher BMI, and when evaluated for the study exhibited, on average, severe depressive symptoms and moderate manic symptoms (Table 1).

\subsection{Sleep and inflammation variables}

The HC group slept an average of 6.9 hours a night, with a standard deviation of about 1 hour; and the mean lag (variability in bedtime from night to night) was 4 minutes, with a standard deviation of 1 hour. The BD group slept an average of 6.5 hours, with a standard deviation of almost 2 hours, and the mean lag was 23 minutes, with a standard deviation of 60 minutes. Total sleep time was correlated positively with depressive symptom severity and negatively with the severity of manic symptoms (Table 2).

\subsection{1 | Group comparisons of TRP, KYN/TRP, and neopterin}

As hypothesized, the BD group had significantly lower levels of TRP than the HC group $(P<.03)$ (Table 1). The BD group did not differ significantly from the $\mathrm{HC}$ group in mean levels of KYN, KYN/TRP, and neopterin (Table 1).

\subsection{2 | TRP, KYN/TRP, and neopterin, and severity of depressive and manic symptoms}

Based on Pearson's correlation test, KYN, KYN/TRP, and neopterin did not correlate significantly with depressive and manic symptoms, as measured by the HDRS and CARS-M clinical measures or sleep
TABLE 1 Demographics, clinical symptoms, and differences in sleep and biomarkers between healthy controls and bipolar disorder individuals

\begin{tabular}{|c|c|c|c|}
\hline & \multirow{2}{*}{$\frac{\text { Healthy control }}{\mathrm{N}(\%)}$} & \multicolumn{2}{|l|}{ Bipolar disorder } \\
\hline & & $N(\%)$ & $P$ \\
\hline Total sample size & 28 & 21 & \\
\hline Gender (F) & $16(57 \%)$ & $10(48 \%)$ & .57 \\
\hline \multicolumn{4}{|l|}{ Race } \\
\hline Asian & $3(11 \%)$ & 0 & .19 \\
\hline Black & $1(4 \%)$ & 0 & \\
\hline White & $24(86 \%)$ & 21 (100\%) & \\
\hline Marital status & & & .02 \\
\hline Married & $14(50 \%)$ & $4(18.2 \%)$ & \\
\hline Not married & $14(50 \%)$ & 17 (81.8\%) & \\
\hline Employment & & & $<.001$ \\
\hline Unemployed & $2(7 \%)$ & $11(55 \%)$ & \\
\hline Disabled & 0 & $4(20 \%)$ & \\
\hline Employed & $16(57 \%)$ & $4(20 \%)$ & \\
\hline Student & $10(36 \%)$ & $1(5 \%)$ & \\
\hline \multirow[t]{2}{*}{ Smoking status } & $1(4 \% /)$ & $11(52 \%)$ & $<.001$ \\
\hline & Mean (SD) & Mean (SD) & \\
\hline Age (y) & $31.57(10.33)$ & $36.10(11.33)$ & .16 \\
\hline $\begin{array}{l}\text { Body mass } \\
\text { index }\left(\mathrm{kg} / \mathrm{m}^{2}\right)\end{array}$ & 25.01 (4.91) & $30.32(5.49)$ & .001 \\
\hline Mania (CARS-M) & 0 & $16.52(13.87)$ & $<.001$ \\
\hline Depression (H-21) & $0.32(.61)$ & 33.62 (15.37) & $<.001$ \\
\hline $\begin{array}{l}\text { Total sleep time } \\
\text { (min) }\end{array}$ & 414.68 (70.98) & 392.56 (117.23) & .45 \\
\hline $\begin{array}{l}\text { Bedtime variability } \\
\text { (min) }\end{array}$ & $-3.67(58.31)$ & $-22.62(61.17)$ & .26 \\
\hline $\mathrm{KYN}(\mu \mathrm{mol} / \mathrm{L})$ & $1.98(.55)$ & $1.79(.50)$ & .22 \\
\hline $\operatorname{TRP}(\mu \mathrm{mol} / \mathrm{L})$ & $68.74(11.62)$ & 60.25 (14.30) & .03 \\
\hline KYN/TRP & $28.93(7.26)$ & $29.88(6.21)$ & .63 \\
\hline Neopterin (nmol/L) & 5.45 (1.39) & $5.80(1.66)$ & .46 \\
\hline
\end{tabular}

KYN, kynurenine; Kyn/Trp, kynurenine/tryptophan ratio; SD, standard deviation; TRP, tryptophan.

measures, including total sleep time and bedtime variability. None of the kynurenine pathway markers were associated with total sleep time in either the BD or $\mathrm{HC}$ group. Neopterin levels correlated positively with KYN/TRP $(r=.53, P<.01)$ but were not correlated with clinical severity, TRP, or KYN (Table 3 ).

\subsubsection{Association of biomarkers and sleep with depressive and manic severity}

The association of depressive severity was tested with four hierarchical regression models, using depressive severity as the dependent variable and total sleep time, KYN, TRP, and KYN/TRP as the predictor variables controlling for BMI (Table 4). Depressive severity was 
TAB LE 2 Bivariate correlations among clinical symptoms of depression, mania, inflammation, and sleep variables in bipolar participants only $(n=21)$

\begin{tabular}{|c|c|c|c|c|c|c|c|c|c|}
\hline & HDRS21 & CARS-M & $\mathrm{BMI}$ & Total sleep time & Mean lag & TRP & KYN & Kyn/Trp & Neopterin \\
\hline HDRS21 & & $-0.62^{* *}$ & 0.11 & $0.72^{* *}$ & -0.32 & 0.36 & 0.44 & 0.14 & -0.11 \\
\hline CARS-M & & & -0.05 & $-0.52^{*}$ & 0.32 & -0.04 & -0.25 & -0.25 & 0.36 \\
\hline Total sleep time & & & & & -0.25 & 0.21 & 0.03 & -0.26 & -0.34 \\
\hline Mean lag & & & & & & -0.05 & 0.21 & 0.33 & 0.25 \\
\hline KYN & & & & & & & & $0.56^{*}$ & 0.24 \\
\hline Kyn/Trp & & & & & & & & & $0.53^{*}$ \\
\hline
\end{tabular}

BMI, body mass index; CARS-M, Clinician-Administered Rating Scale for Mania; HDRS21, Hamilton Depression Rating Scale-21; KYN, kynurenine; Kyn/ $\operatorname{TrP}$, kynurenine/tryptophan ratio; TrP, tryptophan.

${ }^{*} P<.05$

${ }^{* *} P<.01$

TAB LE 3 Bivariate correlations among inflammation and sleep variables in healthy control participants only $(n=28)$

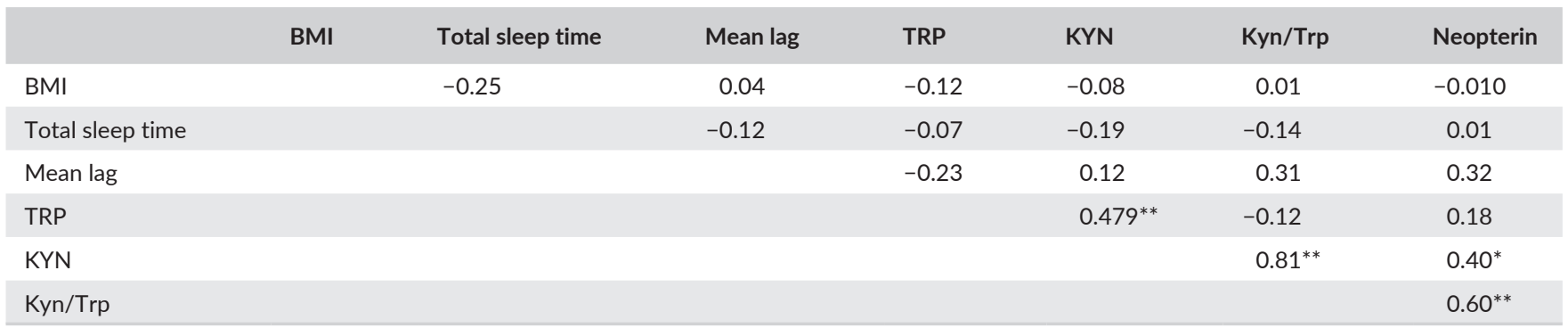

BMI, body mass index; KYN, kynurenine; Kyn/TrP, kynurenine/tryptophan ratio; TrP, tryptophan.

${ }^{*} P<.05$

${ }^{* *} P<.01$

significantly associated with KYN (model 3) and TRP (model 4), when total sleep time and BMI were accounted for. Models 3 and 4 (Table 4) accounted for $71 \%$ and $60 \%$ of the variance, respectively. Model 1 , consisting of KYN/TRP and total sleep time, trended toward significance, accounting for $60 \%$ of the variance (Table 4). Although total sleep time was significantly associated with manic severity in all four models, only the KYN/TRP biomarker trended towards significance (Table 5). This model explained $35 \%$ of the variance (Table 5).

\section{3 | Follow-up data}

Exploratory analysis of the follow-up data showed that although the BD participants reported significantly decreased clinical symptoms of depression and mania, ${ }^{35}$ no significant differences were detected in total sleep time, TRP, KYN/TRP. Total sleep time appears to have increased at follow-up but was not significantly different from baseline scores. This may have been the result of the high variability in total sleep time score for BD participants (Table 6).

\section{4 | DISCUSSION}

The current study examined the relationship between objective markers of sleep and biomarkers of TRP metabolism and inflammation in a group of acutely symptomatic BD individuals. We found significantly lower levels of TRP in BD patients compared to HC. We also found that biomarkers, in conjunction with total sleep time, were associated positively with depressive severity and negatively with manic severity in BD patients.

Our first question addressed whether there were group differences between BD and HC in total sleep time, TRP, and KYN/TRP. Our finding of lower TRP levels in acutely symptomatic BD participants was concordant with several recent reports examining TRP levels in BD and schizophrenia ${ }^{10,39,40}$ and the theory that decreased plasma TRP levels in BD participants may potentially lead to dysfunctional brain serotonin synthesis and release. TRP depletion studies ${ }^{41,42}$ provide a useful tool to examine the role and association of serotonin in different psychiatric disorders. Most of the TRP depletion studies have focused on unipolar depression owing to decreased serotonin as an underlying cause. For example, TRP depletion has been associated either with no effect ${ }^{43}$ or with a slight but insignificant relapse of manic symptoms in fully recovered BD patients. No TRP depletion studies have been carried out in acutely manic BD patients. ${ }^{39}$ The present study provides evidence for decreased TRP levels in a naturalistic occurrence of acute mood episodes. While experimental studies support the notion that decreased levels may be a precursor to a depressed mood state, in our study it was unclear whether the decreased TRP levels preceded or resulted from an acute mood state. 
TABLE 4 Association between depressive symptom severity and total sleep time and KYN/TRP ratio in bipolar disorder (controlling for $\mathrm{BMI})(\mathrm{n}=21)$

\begin{tabular}{|lllll|}
\hline Variable & $\boldsymbol{\beta}$ & $\boldsymbol{t}$ & $\boldsymbol{R}$ & Adj. $\mathbf{R}^{2}$ \\
\hline Model 1 & & & 0.81 & 0.60 \\
\hline BMI & 0.05 & 0.34 & & \\
\hline Total sleep time & 0.85 & $5.42^{* *}$ & & \\
\hline KYN/TRP & 0.32 & $2.0^{\wedge}$ & & \\
\hline Model 2 & & & 0.54 & 0.24 \\
\hline BMI & 0.50 & $3.85^{* *}$ & & \\
\hline Total sleep time & 0.27 & $2.15^{*}$ & & \\
\hline Neopterin & 0.17 & 0.92 & & \\
\hline Model 3 & & & 0.85 & 0.71 \\
\hline BMI & 0.21 & 1.53 & & \\
\hline Total sleep time & 0.78 & $5.88^{* *}$ & & \\
\hline KYN & 0.39 & $2.93^{* *}$ & & \\
\hline Model 4 & & & 0.81 & 0.60 \\
\hline BMI & 0.30 & 1.83 & & \\
\hline Total sleep time & 0.66 & $4.53^{* *}$ & & \\
\hline TRP & 0.35 & $2.10^{*}$ & & \\
\hline
\end{tabular}

BMI, body mass index; Kyn, kynurenine; Kyn/Trp, kynurenine/tryptophan ratio; TRP, tryptophan.

${ }^{*} P<.05$

${ }^{* *} P<.01$

$\wedge=0.06$

TABLE 5 Association between manic symptom severity and total sleep time and KYN/TRP in the bipolar disorder group (controlling for BMI) $(n=21)$

\begin{tabular}{|l|llll|}
\hline Variable & $\boldsymbol{\beta}$ & $\boldsymbol{t}$ & $\boldsymbol{R}$ & Adj. $^{2}$ \\
\hline Model 1 & & & 0.66 & 0.35 \\
\hline BMI & 0.04 & 0.19 & & \\
\hline Total sleep time & -0.69 & $-3.59^{*}$ & & \\
\hline KYN/TRP & -0.39 & $-2.00^{* *}$ & & \\
\hline Model 2 & & & 0.40 & 0.10 \\
\hline BMI & 0.17 & 1.21 & & \\
\hline Total sleep time & -0.29 & $-2.12^{*}$ & & \\
\hline Neopterin & 0.12 & 0.90 & & \\
\hline Model 3 & & & 0.60 & 0.25 \\
\hline BMI & -0.21 & -0.57 & & \\
\hline Total sleep time & -0.57 & $-2.97^{*}$ & & \\
\hline KYN & -0.21 & -1.07 & & \\
\hline Model 4 & & & 0.57 & 0.21 \\
\hline BMI & -0.06 & -0.28 & & \\
\hline Total sleep time & -0.56 & $-2.76^{*}$ & & \\
\hline TRP & 0.05 & 0.20 & & \\
\hline
\end{tabular}

BMI, body mass index; Kyn, kynurenine; Kyn/TrP, kynurenine/tryptophan ratio; Trp, tryptophan.

${ }^{*} P<.05$

${ }^{* *} P=.06$
Secondly, we asked if TRP, KYN/TRP, and neopterin were associated with severity of depressive and manic symptoms. We did not find levels of neopterin, an inflammatory marker, to be significantly different from those in $\mathrm{HC}$, and they were not associated with depression or mania severity. However, neopterin levels were found to be associated positively with the KYN/TRP ratio, which replicated earlier studies investigating depression and chronic inflammation. ${ }^{44,45}$ Further mechanistic studies examining the role of neopterin in mood disorders are warranted. For example, one study ${ }^{25}$ found that significantly higher levels of neopterin predicted poststroke depression in patients who had previously experienced a major depressive episode. Another crosssectional study found a positive association between MDD with a history of suicide attempt and KYN but no association with neopterin. ${ }^{44}$ However, similar to our findings, Sublette et $\mathrm{al}^{44}$ found a positive correlation between the cytokine activation marker neopterin and KYN/ TRP, suggesting that KYN production may be influenced by inflammatory processes. The present study supports previous studies finding an association between neopterin and the KYN/TRP ratio but does not support a direct association between neopterin and mood disorders.

We hypothesized a significant association between sleep duration and biomarkers (TRP, KYN/TRP, neopterin), and depressive and manic symptom severity. TRP degradation follows one of two main metabolic pathways, which ultimately lead to the production of either serotonin and melatonin, or kynurenine and its metabolites (Figure 1). Enhanced activation of the kynurenine pathway due to stress or an acute mood state would theoretically hinder melatonin production, thereby potentially affecting the sleep patterns of patients. ${ }^{6,46,47}$ Thus, activation of the kynurenine pathway potentially suggests the association of sleep and the kynurenine metabolites with mood states. While we did not find a correlation between these biomarkers and severity of mood symptoms, we found an association with the severity of clinical mood symptoms when considering KYN/TRP in conjunction with objective total sleep time. Neopterin and TRP degradation, as measured by the KYN/ TRP, is highly associated with immune activation, which in turn leads to the production of KYN instead of serotonin from TRP; ${ }^{48}$ however, in our data neopterin, although correlated with KYN/TRP, was not associated with mood severity, with or without accounting for sleep. The association of KYN, TRP, and total sleep time with severity of depression and manic symptoms implies that these pathways are related but further research is necessary to delineate exactly how they influence each other.

Finally, we asked whether total sleep time, TRP, and KYN/TRP changed with symptom remission in asymptomatic BD patients. Despite subjectively reported decreased clinical symptoms, no differences were detected in the biomarkers or total sleep time. One explanation could be the short follow-up time - perhaps these markers change on a slower time scale, or they may leave a long-term residual mark of the episode, indicating a change in physiology despite remission. ${ }^{49-51}$

However, the results should be treated with caution owing to the small sample size of the study. Additionally, the patients were all receiving medication for an acute mood episode, which makes it difficult to parse the effects of medication. Further, in order to maximize statistical power, the study investigated only one sleep variable - total 
TABLE 6 Paired sample $t$ test comparing clinical symptoms of depression, mania, sleep, and inflammation variables at baseline and follow-up in bipolar disorder Data represents mean (SD)

\begin{tabular}{|lcccc} 
& Baseline & Follow-up & N & P-value \\
\hline Mania & $19.69(15.11)$ & $3.75(5.47)$ & 16 & $<.001$ \\
\hline $\begin{array}{l}\text { Depression } \\
\begin{array}{l}\text { Total sleep time } \\
(\mathrm{min})\end{array}\end{array}$ & $32.40(14.32)$ & $11.13(10.60)$ & 15 & $<.001$ \\
\hline $\mathrm{KYN}(\mu \mathrm{mol} / \mathrm{L})$ & $353.34(124.68)$ & $421.51(125.70)$ & 8 & .24 \\
\hline $\mathrm{TRP}(\mu \mathrm{mol} / \mathrm{L})$ & $1.69(.48)$ & $1.70(0.50)$ & 13 & .92 \\
\hline $\mathrm{KYN} / \mathrm{TRP}$ & $61.24(15.91)$ & $62.04(13.78)$ & 13 & .82 \\
\hline
\end{tabular}

Kyn, kynurenine; Kyn/TrP, kynurenine/tryptophan ratio; Trp, tryptophan. sleep time. However, sleep is a complex phenomenon and consists of multiple aspects which are easily measured using actigraphic devices. Based on the results of the present study, different sleep variables, along with inflammatory markers, warrant further investigation with a larger sample size. Another important methodological factor was the use of a structured hospitalized setting compared to a home environment. It may be argued that a hospitalized setting led to patients feeling more stressed than the HC; however, we argue that the patients were already in acute distress and unable to cope with their existing resources, leading to inpatient hospitalization. The structured setting of the hospital often relieves stress through learning basic skills and teaching patients to generalize those skills to real-world naturalistic settings. $^{52}$

\section{5 | CONCLUSION}

In conclusion, the study provides evidence supporting the association of mood severity in acutely symptomatic BD participants with metabolites of the KYN pathway, in association with objective measurements of sleep. Further understanding of the role of in BD is important for the development of potential targets for intervention, or markers of treatment response. Targeting sleep and regulation of the TRP breakdown pathway for intervention in BD participants is an area of research warranting further investigation. Targeting these areas for improvement and observing clinically significant changes may lead to more sustained improvement and remission rates.

\section{ACKNOWLEDGEMENTS}

The authors thank the participants and families for generously being part of this project. The authors acknowledge Penn State Clinical \& Translational Research Institute, Pennsylvania State University for funding this project. Study data were collected and managed using REDCap (Research Electronic Data Capture) electronic data capture tools hosted at the Penn State Milton S. Hershey Medical Center and College of Medicine.

\section{DISCLOSURES}

The project described was supported by the National Center for Advancing Translational Sciences, Grant KL2 TR000126. The content is solely the responsibility of the authors and does not necessarily represent the official views of the NIH. Dr. Postolache's contribution was supported by a Distinguished Investigator Award (DIG-1162-12) from the American Association for Suicide Prevention by the Rocky Mountain MIRECC, the VA Merit Review CSR\&D grant 1 I01 CX001310-01A1 and the Joint Institute for Food Safety and Applied Nutrition/UMD through the cooperative agreement FDU.001418.

\section{REFERENCES}

1. Goodwin FK, Jamison KR. Manic-Depressive Illness: Bipolar Disorders and Recurrent Depression. New York, NY: Oxford University Press; 2007

2. Cusin C, Serretti A, Lattuada E, Mandelli L, Smeraldi E. Impact of clinical variables on illness time course in mood disorders. Psychiatry Res. 2000;97:217-227.

3. Manji HK, Lenox RH. Signaling: cellular insights into the pathophysiology of bipolar disorder. Biol Psychiat. 2000;48:518-530.

4. Manji HK, Quiroz JA, Payne JL, et al The underlying neurobiology of bipolar disorder. World Psychiatry. 2003;2:136.

5. Leboyer M, Soreca I, Scott J, et al Can bipolar disorder be viewed as a multi-system inflammatory disease? J Affect Disord. 2012;141:1-10.

6. Anderson G, Jacob A, Bellivier F, Geoffroy P. Bipolar disorder: the role of the kynurenine and melatonergic pathways. Curr Pharm Des. 2015;22:987-1012.

7. Berk M, Kapczinski F, Andreazza A, et al Pathways underlying neuroprogression in bipolar disorder: focus on inflammation, oxidative stress and neurotrophic factors. Neurosci Biobehav Rev. 2011;35:804-817.

8. Harvey AG, Schmidt DA, Scarnà A, Semler CN, Goodwin GM. Sleeprelated functioning in euthymic patients with bipolar disorder, patients with insomnia, and subjects without sleep problems. Am J Psychiatry. 2005;162:50-57.

9. Miller CL, Llenos IC, Dulay JR, Weis S. Upregulation of the initiating step of the kynurenine pathway in postmortem anterior cingulate cortex from individuals with schizophrenia and bipolar disorder. Brain Res. 2006;1073:25-37.

10. Clark SM, Pocivavsek A, Nicholson JD, et al Reduced kynurenine pathway metabolism and cytokine expression in the prefrontal cortex of depressed individuals. J Psychiatry Neurosci. 2016;41:368-394.

11. Murray G, Harvey A. Circadian rhythms and sleep in bipolar disorder. Bipolar Disord. 2010;12:459-472.

12. Harvey AG, Soehner AM, Kaplan KA, et al Treating insomnia improves mood state, sleep, and functioning in bipolar disorder: a pilot randomized controlled trial. J Consult Clin Psychol. 2015;83:564.

13. Kanady JC, Soehnera AM, Harvey AG. A retrospective examination of sleep disturbance across the course of bipolar disorder. J Sleep Disord Ther. 2015;4:193. https://doi.org/10.4172/2167-0277.1000193

14. Goldstein BI, Kemp DE, Soczynska JK, McIntyre RS. Inflammation and the phenomenology, pathophysiology, comorbidity, and treatment 
of bipolar disorder: a systematic review of the literature. J Clinical Psychiatry. 2009;70:1078-1090.

15. Myint AM, Kim Y-K, Verkerk R, et al Tryptophan breakdown pathway in bipolar mania. J Affect Disord. 2007;102:65-72.

16. Fernandez-Mendoza J, Vgontzas AN. Insomnia and its impact on physical and mental health. Curr Psychiatry Rep. 2013;15:1-8.

17. Gaines J, Vgontzas AN, Fernandez-Mendoza J, Kritikou I, Basta M, Bixler EO. Gender differences in the association of sleep apnea and inflammation. Brain Behav Immun. 2015;47:211-217.

18. Etain B, Milhiet V, Bellivier F, Leboyer M. Genetics of circadian rhythms and mood spectrum disorders. Eur Neuropsychopharmacol. 2011;21:S676-S682.

19. Saunders EF, Novick DM, Fernandez-Mendoza J, et al Sleep quality during euthymia in bipolar disorder: the role of clinical features, personality traits, and stressful life events. Int J Bipolar Disord. 2013;1:16.

20. Saunders EF, Fernandez-Mendoza J, Kamali M, Assari S, McInnis MG The effect of poor sleep quality on mood outcome differs between men and women: a longitudinal study of bipolar disorder. J Affect Disord. 2015;180:90-96.

21. Geoffroy P, Scott J, Boudebesse C, et al Sleep in patients with remitted bipolar disorders: a meta-analysis of actigraphy studies. Acta Psychiatr Scand. 2015;131:89-99.

22. Gaines J, Vgontzas AN, Fernandez-Mendoza J, et al Inflammation mediates the association between visceral adiposity and obstructive sleep apnea in adolescents. Am J Physiol Endocrinol Metab. 2016;311:E851-E858.

23. Li Y, Vgontzas AN, Fernandez-Mendoza J, et al Objective, but not subjective, sleepiness is associated with inflammation in sleep apnea. Sleep. 2017; pii:sp-00209-16.

24. Fernandez-Mendoza J, Baker JH, Vgontzas AN, Gaines J, Liao D, Bixler EO. Insomnia symptoms with objective short sleep duration are associated with systemic inflammation in adolescents. Brain Behav Immun. 2017;61:110-116.

25. Tang C-Z, Zhang Y-L, Wang W-S, Li W-G, Shi J-P. Elevated serum levels of neopterin at admission predicts depression after acute ischemic stroke: a 6-month follow-up study. Mol Neurobiol. 2015;53:1-11.

26. Schröcksnadel K, Widner B, Bergant $A$, et al Longitudinal study of tryptophan degradation during and after pregnancy. Life Sci. 2003;72:785-793.

27. Schröcksnadel H, Baier-Bitterlich G, Dapunt O, Wachter H, Fuchs D. Decreased plasma tryptophan in pregnancy. Obstet Gynecol. 1996;88:47-50

28. Fuchs D, Hausen A, Reibnegger G, Werner ER, Dierich MP, Wachter H. Neopterin as a marker for activated cell-mediated immunity: application in HIV infection. Immunol Today. 1988;9:150-155.

29. Schwarcz R, Whetsell WO, Mangano RM. Quinolinic acid: an endogenous metabolite that produces axon-sparing lesions in rat brain. Science. 1983;219:316-318.

30. Stone T, Perkins M. Quinolinic acid: a potent endogenous excitant at amino acid receptors in CNS. Eur J Pharmacol. 1981;72:411-412.

31. Tavares RG, Tasca Cl, Santos CE, et al Quinolinic acid stimulates synaptosomal glutamate release and inhibits glutamate uptake into astrocytes. Neurochem Int. 2002;40:621-627.

32. Sheehan DV, Lecrubier $Y$, Sheehan KH, et al The Mini-International Neuropsychiatric Interview (M.I.N.I.): the development and validation of a structured diagnostic psychiatric interview for DSM-IV and ICD10. J Clin Psychiatry. 1998;59(Suppl. 20):22-33.

33. Hamilton M. A rating scale for depression. J Neurol Neurosurg Psychiatry. 1960;23:56-62.

34. Altman EG, Hedeker DR, Janicak PG, Peterson JL, Davis JM. The Clinician-Administered Rating Scale for Mania (CARS-M): development, reliability, and validity. Biol Psychiatry. 1994;36:124-134.
35. Saunders EF, Reider A, Singh G, Gelenberg AJ, Rapoport SI. Low unesterified: esterified eicosapentaenoic acid (EPA) plasma concentration ratio is associated with bipolar disorder episodes, and omega-3 plasma concentrations are altered by treatment. Bipolar Disord. 2015;17:729-742.

36. Laich A, Neurauter G, Widner B, Fuchs D. More rapid method for simultaneous measurement of tryptophan and kynurenine by HPLC. Clin Chem. 2002;48:579-581.

37. Widner B, Werner ER, Schennach H, Wachter H, Fuchs D. Simultaneous measurement of serum tryptophan and kynurenine by HPLC. Clin Chem. 1997;43:2424-2426.

38. Jung UJ, Choi M-S. Obesity and its metabolic complications: the role of adipokines and the relationship between obesity, inflammation, insulin resistance, dyslipidemia and nonalcoholic fatty liver disease. Int $J$ Mol Sci. 2014;15:6184-6223.

39. Bell C, Abrams J, Nutt D. Tryptophan depletion and its implications for psychiatry. Br J Psychiatry. 2001;178:399-405.

40. Chiappelli J, Postolache TT, Kochunov P, et al Tryptophan metabolism and white matter integrity in schizophrenia. Neuropsychopharmacology. 2016;41:2587-2595.

41. Benkelfat C, Seletti B, Palmour RM, Hillel J, Ellenbogen M, Young SN Tryptophan depletion in stable lithium-treated patients with bipolar disorder in remission. Arch Gen Psychiatry. 1995;52:154-155.

42. Booij L, Van der Does W, Benkelfat C, et al Predictors of mood response to acute tryptophan depletion: a reanalysis. Neuropsychopharmacology. 2002;27:852-861.

43. Cassidy F, Murry E, Carroll BJ. Tryptophan depletion in recently manic patients treated with lithium. Biol Psychiat. 1998;43:230-232.

44. Sublette ME, Galfalvy HC, Fuchs D, et al Plasma kynurenine levels are elevated in suicide attempters with major depressive disorder. Brain Behav Immun. 2011;25:1272-1278.

45. Schröcksnadel K, Wirleitner B, Winkler C, Fuchs D. Monitoring tryptophan metabolism in chronic immune activation. Clin Chim Acta. 2006;364:82-90.

46. Brzezinski A, Vangel MG, Wurtman RJ, et al Effects of exogenous melatonin on sleep: a meta-analysis. Sleep Med Rev. 2005;9:41-50.

47. Dijk D-J, Cajochen C. Melatonin and the circadian regulation of sleep initiation, consolidation, structure, and the sleep EEG. J Biol Rhythms. 1997;12:627-635

48. Widner B, Laich A, Sperner-Unterweger B, Ledochowski M, Fuchs D. Neopterin production, tryptophan degradation, and mental depression - what is the link? Brain Behav Immun. 2002;16:590-595.

49. Benkelfat C, Ellenbogen MA, Dean P, Palmour RM, Young SN. Moodlowering effect of tryptophan depletion: enhanced susceptibility in young men at genetic risk for major affective disorders. Arch Gen Psychiatry. 1994;51:687-697.

50. Van der Does AW. The effects of tryptophan depletion on mood and psychiatric symptoms. J Affect Disord. 2001;64:107-119.

51. Riedel WJ, Klaassen T, Schmitt JA. Tryptophan, mood, and cognitive function. Brain Behav Immun. 2002;16:581-589.

52. Björgvinsson T, Kertz SJ, Bigda-Peyton JS, Rosmarin DH, Aderka IM, Neuhaus EC. Effectiveness of cognitive behavior therapy for severe mood disorders in an acute psychiatric naturalistic setting: A benchmarking study. Cogn Behav Ther. 2014;43:209-220.

How to cite this article: Mukherjee D, Krishnamurthy VB, Millett CE, Reider A, Can A, Groer M, Fuchs D, Postolache TT, Saunders $\mathrm{EFH}$. Total sleep time and kynurenine metabolism associated with mood symptom severity in bipolar disorder. Bipolar Disorders. 2018;20:27-34. https://doi.org/10.1111/bdi.12529 